\title{
The polycomb group protein BMI-1 inhibitor PTC-209 is a potent anti-myeloma agent alone or in combination with epigenetic inhibitors targeting EZH2 and the BET bromodomains
}

\author{
Mohammad Alzrigat ${ }^{1}$, Alba Atienza Párraga ${ }^{1}$, Muntasir Mamun Majumder ${ }^{2}$ Anqi \\ $\mathrm{Ma}^{3}$, Jian $\mathrm{Jin}^{3}$, Anders Österborg ${ }^{4}$, Hareth $\mathrm{Nahi}^{5}$, Kenneth Nilsson ${ }^{1}$, Caroline A. \\ Heckmann $^{2}$, Fredrik Öberg ${ }^{1}$, Antonia Kalushkova ${ }^{1}$ and Helena Jernberg-Wiklund ${ }^{1}$ \\ ${ }^{1}$ Science for Life Laboratory, Department of Immunology, Genetics and Pathology, Rudbeck Laboratory, Uppsala University, \\ Uppsala, Sweden \\ ${ }^{2}$ Institute for Molecular Medicine Finland (FIMM), Helsinki Institute of Life Science, University of Helsinki, Helsinki, Finland \\ ${ }^{3}$ Departments of Pharmacological Sciences and Oncological Sciences, Icahn School of Medicine at Mount Sinai, New York, \\ NY, USA \\ ${ }^{4}$ Department of Oncology-Pathology, Karolinska University Hospital, Solna, Stockholm, Sweden \\ ${ }^{5}$ Department of Medicine, Unit of Hematology, Karolinska University Hospital, Huddinge, Stockholm, Sweden \\ Correspondence to: Helena Jernberg-Wiklund, email: helena.jernberg_wiklund@igp.uu.se \\ Mohammad Alzrigat, email: mohammad.alzrigat@igp.uu.se
}

Keywords: multiple myeloma, epigenetics, polycomb, BMI-1, PTC-209

Received: February 14, $2017 \quad$ Accepted: October 10, 2017

Published: October 20, 2017

Copyright: Alzrigat et al. This is an open-access article distributed under the terms of the Creative Commons Attribution License 3.0 (CC BY 3.0), which permits unrestricted use, distribution, and reproduction in any medium, provided the original author and source are credited.

\section{ABSTRACT}

Multiple myeloma (MM) is a tumor of plasmablasts/plasma cells (PCs) characterized by the expansion of malignant PCs with complex genetic aberrations in the bone marrow (BM). Recent reports, by us and others, have highlighted the polycomb group (PcG) proteins as potential targets for therapy in MM. The PcG protein BMI-1 of the polycomb repressive complex 1 (PRC1) has been reported to be overexpressed and to possess oncogenic functions in MM. Herein, we report on the anti-myeloma effects of the BMI-1 inhibitor PTC-209 and demonstrate that PTC-209 is a potent anti-myeloma agent in vitro using MM cell lines and primary MM cells. We show that PTC-209 reduces the viability of MM cells via induction of apoptosis and reveal that the anti-MM actions of PTC-209 are mediated by on-target effects i.e. downregulation of BMI-1 protein and the associated repressive histone mark H2AK119ub, leaving other PRC1 subunits such as CBX-7 and the catalytic subunit RING1B unaffected. Importantly, we demonstrate that PTC-209 exhibits synergistic and additive anti-myeloma activity when combined with other epigenetic inhibitors targeting EZH2 and BET bromodomains. Collectively, these data qualify BMI-1 as a candidate for targeted therapy in MM alone or in combinations with epigenetic inhibitors directed to PRC2/EZH2 or BET bromodomains.

\section{INTRODUCTION}

Multiple myeloma (MM) is a genetically complex and heterogeneous disease characterized by abnormal proliferation of clonal plasmablasts/plasma cells (PCs) in the bone marrow (BM) [1-3]. Current treatment strategies in MM such as the use of proteasome inhibitors (e.g. bortezomib) and immunomodulatory drugs (e.g. thalidomide and lenalidomide) have improved the outcome of patients [4]. However, MM remains a therapeutically challenging malignancy due to the high rate of relapse and the development of drug resistance with a median survival time of less than 5 years [5]. The current view suggests that a combination of genetic and epigenetic aberrations accompanied by growth support from the tumor microenvironment in the bone marrow are important factors mediating drug resistance and relapse in $\mathrm{MM}$ $[6,7]$. 
Epigenetic mechanisms play important roles in fundamental biological processes such as maintenance of pluripotency, cellular differentiation and cell reprogramming [8, 9], and aberrations in epigenetic modifiers and mediators have been implicated in tumor initiation and progression [10-12]. Supporting the importance of epigenetic mechanisms in MM pathogenesis, genetic alterations affecting components of epigenetic machineries i.e. epigenetic modifiers, mediators and substrates such as histone proteins, have been reported in MM [13-15]. In addition, deregulated expression and function of epigenetic modifiers such as the enhancer of zeste homolog 2 (EZH2) [16-18], the multiple myeloma SET domain containing-protein (MMSET) $[19,20]$ and members of the Jumonji C-domain-containing histone demethylases KDM6B [21] and KDM3A [22] are frequently observed in MM. Collectively, these data point towards a potential role of epigenetic reprogramming in $\mathrm{MM}$, and highlight epigenetic modifiers as promising therapeutic targets in MM.

The polycomb group protein B Lymphoma MoMLV Insertion Region 1 (BMI-1) is a core subunit of the polycomb repressive complex 1 (PRC1), which plays important roles during normal development and tumorigenesis $[23,24]$. PRC1 mediates gene repression via mono-ubiquitination of lysine 119 on histone H2A (H2AK119ub) and subsequent chromatin compaction $[25,26]$. BMI-1 is involved in the regulation of important biological processes such as cell cycle, DNA damage response, stemness and senescence [27]. BMI1 was initially discovered as a co-factor of c-MYC in lymphomagenesis [28, 29]. Later on, BMI-1 has been described as an oncogene in a wide range of solid and hematopoietic tumors [27, 30]. Notably, BMI-1 has been shown to mediate the growth and survival of cancer stem cells in solid and hematological malignancies [31-33]. Recently, the development of PTC-209, a small specific inhibitor of BMI-1, has opened new avenues to evaluate the therapeutic potential of BMI-1 in cancer including MM [31, 32, 34, 35]. BMI-1 was recently found to be overexpressed in MM compared to normal PCs [36-38] and to promote $\mathrm{MM}$ cells growth in vitro and in vivo [37]. Recent analyses of gene expression profiling in MM have revealed the overexpression of BMI-1 in all stages of MM progression as compared with normal bone marrow PCs [34]. In addition, high expression of BMI-1 was suggested as a predictor of poor survival in relapsed MM cases treated with bortezomib or dexamethasone [34]. Collectively, these data suggest an important role for BMI-1 in MM pathogenesis and response to treatment and highlight BMI-1 as a potential target for therapy.

In this study, we provide data further emphasizing BMI-1 as a potential therapeutic target in MM using PTC-209. We show that PTC-209 is indeed a potent antiMM agent by reducing the viability of MM cell lines and primary MM cells from newly diagnosed or relapsed patients. We report that these effects are mediated by ontarget effects by reducing the BMI-1 protein levels and the global level of the associated H2AK119ub. Importantly, PTC-209 did not affect other PRC1 subunits such as CBX7 and, especially, the catalytic subunit RING1B. We also present PTC-209 as a promising combinatorial agent with specific epigenetic inhibitors targeting the polycomb group protein EZH2 and BET bromodomains.

\section{RESULTS}

\section{The BMI-1 inhibitor PTC-209 is a potent anti- myeloma agent in vitro}

The reports on BMI-1 overexpression in MM and the development of PTC-209 as a specific inhibitor of BMI-1 prompted us to evaluate the therapeutic potential of targeting BMI-I by using PTC-209 in in vitro models of MM. To this end, we assessed the effects of PTC-209 treatment on the viability of MM cell lines and primary cells. PTC-209 exhibited a potent anti-myeloma activity, reducing the viability of $\mathrm{MM}$ cell lines at concentrations ranging up to $1.6 \mu \mathrm{M}$ over 48 hours of treatment (Figure 1A). PTC-209-mediated reduction of cell viability was variable with INA-6 being the most responsive and U2661970 being the least responsive cell line (Figure 1A). Notably, PTC-209 reduced the viability of MM cell lines tested in this study as early as 24 hours post-treatment (Supplementary Figure 1). To further investigate the anti-MM effects of PTC-209, we tested the effects on the viability of $\mathrm{CD} 138^{+}$malignant PCs purified from newly diagnosed (patients 1-4) or relapsed patients (patients 5-11) in response to 72 hours treatment with a range of PTC-209 concentrations (Figure 1B). We found that a high concentration $(10 \mu \mathrm{M})$ of PTC-209 reduced the viability of all primary MM cells (Figure 1B). Interestingly, the response of malignant CD $138^{+}$PCs to $1 \mu \mathrm{M}$ of PTC-209 identified 3 groups of patients: non-responsive, moderate responders and high responders (Figure 1B). The effect of PTC-209 in reducing the viability of primary MM cells in this study was independent of the disease diagnosis i.e. newly diagnosed or refractory as well as the cytogenetic karyotype of the patients (Supplementary Table 1).

\section{PTC-209 induces apoptosis in MM cells}

To further decipher the anti-MM potential of PTC209, we evaluated the effects of PTC-209 treatment $(0.8$ $\mu \mathrm{M})$ for 48 hours on cell cycle and apoptosis induction by flow cytometry in three of the MM cell lines: INA-6, RPMI-8226 and JJN3. Cell cycle analysis revealed that PTC-209 treatment did not affect the distribution of the cells in different phases of the cell cycle, but induced a significant increase in the percentage of apoptotic cells in the sub-G1 phase (Figure 1C and Supplementary Figure 2). The increase in the percentage of sub-G1 
apoptotic cells upon $0.8 \mu \mathrm{M}$ treatment with PTC-209 varied between cell lines i.e. 5-fold in INA-6 (3 to 15\%), 2.5-fold in RPMI-8226 (14 to 35\%) and 4-fold in JJN3 (5-23\%). To specifically study markers of apoptosis, we evaluated by flow cytometry Annexin V and PI positive cells in control DMSO or PTC-209 $(0.8 \mu \mathrm{M})$ treated MM cell lines. We found that PTC-209 significantly increased the percentage of Annexin $\mathrm{V}$ and PI positive MM cells as compared to DMSO control treatment (Figure 1D and Supplementary Figure 3). The increase in Annexin V/ PI-positive cells due to treatment with PTC-209 varied between cell lines i.e. 4-fold in INA-6 (19.2 to $77.7 \%$ ), 4-fold in RPMI-8226 (18.3 to 73.8\%) and 5-fold in JJN3
(14.2 to $74.1 \%$ ). We further confirmed the apoptotic effects of PTC-209 in MM cell lines by showing increased caspase-3/7 activity (Figure 1E) and induced expression of the pro-apoptotic gene BIM at the mRNA level (Figure 1F).

\section{PTC-209 demonstrates on-target effects in MM}

PTC-209 was initially selected for its capacity to reduce BMI-1 protein expression and to subsequently inactivate PRC1 activity as represented by a decrease in the global levels of H2AK119ub without affecting other subunits of the PRC1 complex [31]. To investigate the
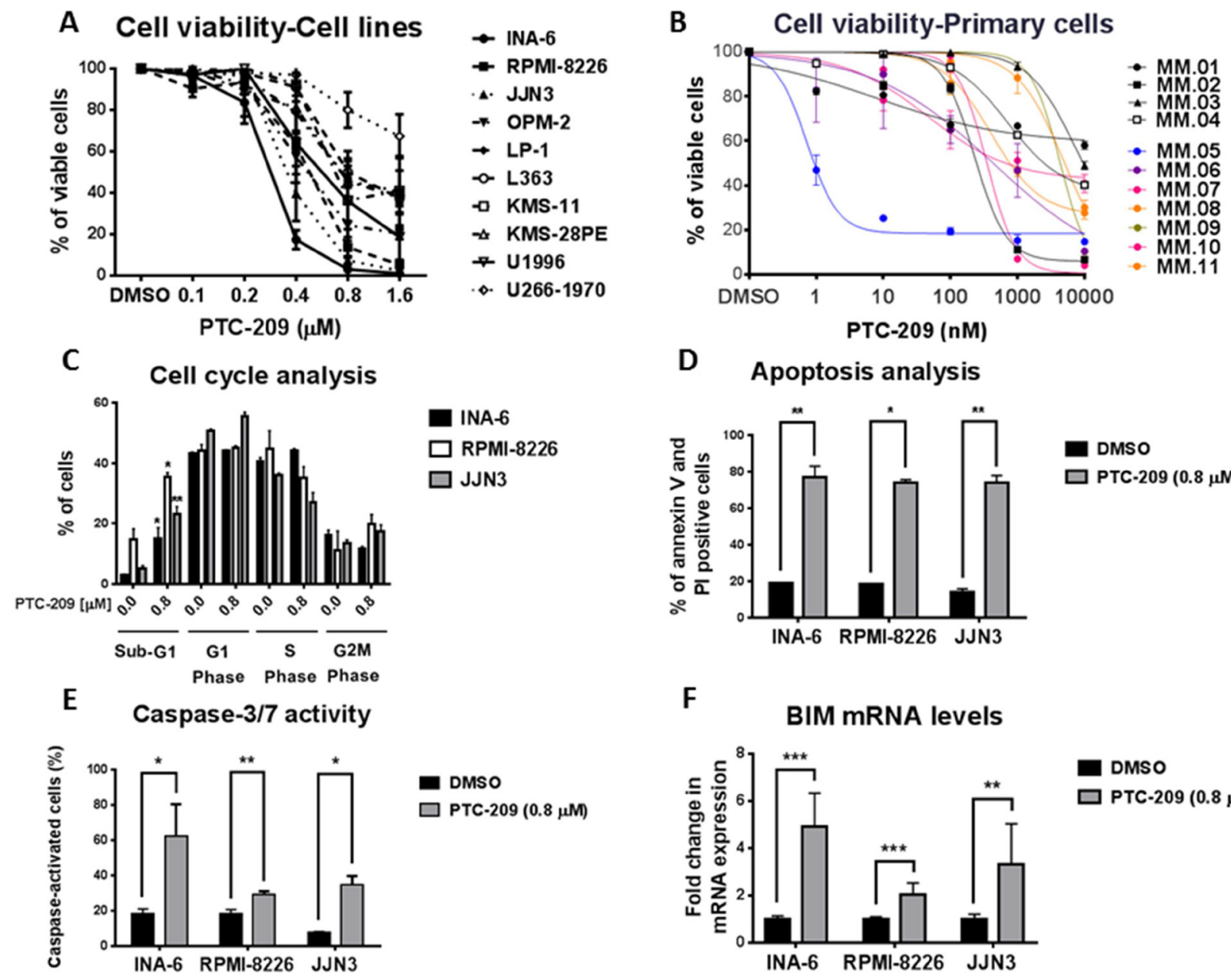

\section{Apoptosis analysis}
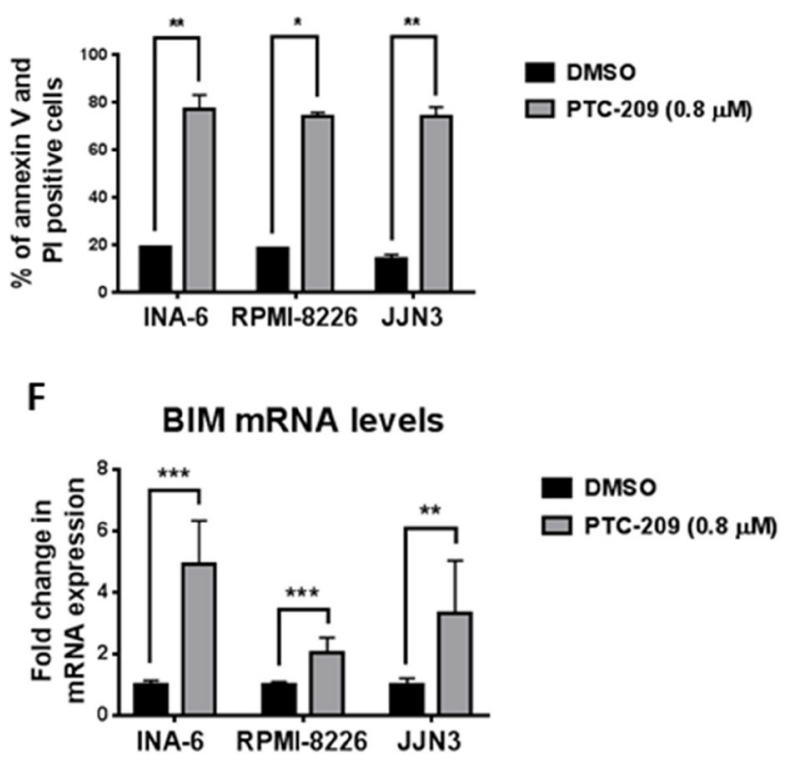

Figure 1: PTC-209 is a potent anti-myeloma agent that induces apoptosis. (A) PTC-209 reduces the viability of MM cell lines. MM cells were treated with a range of PTC-209 concentrations for 48 hours. DMSO was used as control treatment. Cell viability was assessed using AlamarBlue assay. (B) PTC-209 reduces the viability of primary MM cells isolated from newly diagnosed or relapsed MM patients. Cell viability was represented as relative to DMSO-treated sample. Cell viability was measured by CellTiter-Glo assay 72 hours after treatment. (C-F) PTC-209 induces apoptosis in MM, which is evident by the accumulation of cells in sub-G1 phase (C) and an increase in the percentage of Annexin V and PI positive cells (D). PTC-209 mediated induction of apoptosis is further confirmed by the increase in caspase-3/7 activity (E) and induced expression of the pro-apoptotic gene BIM (F). Cell cycle was analyzed by measuring the DNA content using propidium iodide (PI) staining, while apoptosis was measured by assessing percentage of Annexin V and PI positive cells and caspase-3/7 activity. The impact of PTC-209 treatment on BIM expression was evaluated by RT-qPCR analysis of mRNA levels. Actin was used as the reference gene. Cells were treated for 48 hours with $0.8 \mu \mathrm{M}$ PTC-209 or DMSO as control. Error bars represent standard deviation of three independent biological experiments from MM cell lines and three technical replicates for primary cells. $P$-values were calculated using student $t$-test in GraphPad Prism, $P$-value: ${ }^{*} \leq 0.05 ;{ }^{* *} \leq 0.01 ;{ }^{* * *} \leq 0.001$. 
on-target effects of PTC-209 in MM, we evaluated the impact of PTC-209 treatment on BMI-1 expression and the global levels of H2AK119ub in several MM cell lines. By reverse transcription quantitative real-time PCR (RT-qPCR) we found that PTC-209 treatment did not reduce the expression of BMI-1 at the mRNA levels, but rather led to an increase in BMI-1 transcript levels in most of the MM cell lines tested in this study (Figure 2A). Importantly, western blot analysis of BMI-1 protein revealed that PTC-209 treatment downregulated BMI1 protein levels (Figure 2B). The downregulation of BMI-1 protein as a consequence of PTC-209 treatment was accompanied by a reduction in the global levels of the H2AK119ub histone mark mediated by the PRC1 complex (Figure 2B). We further documented the ontarget effects of PTC-209 in MM by showing that other PRC1 subunits i.e. CBX-7 and, most importantly, the E3 ubiquitin ligase RING1B were not affected by the PTC209 treatment (Figure 2B).

\section{PTC-209 demonstrates synergistic and additive activity with UNC1999 and JQ1 in MM cell lines}

Having shown potent anti-myeloma activity for PTC-209, we sought to investigate whether PTC-209 represents a potent anti-MM agent in combination with other epigenetic inhibitors. The possibility of inhibiting both polycomb repressive complexes i.e. PRC1 and PRC2 is highly attractive and supported by the growing evidences showing possible collaboration between the complexes in gene silencing [39-41]. In addition, we and others have recently demonstrated that targeted inhibition of the PRC2 enzymatic subunit EZH2 by highly selective inhibitors had anti-myeloma effects
[42-46]. To this end, we combined PTC-209 with the EZH2 inhibitor UNC1999 and assessed the effects of single or combinatorial treatments on the viability of the MM cell lines INA-6, JJN3, RPMI-8226 and LP-1. We found that combinations of PTC-209 and UNC1999 induced a significant reduction in cell viability when compared to single agent treatment in all cell lines tested in this study as shown in Figure 3A for some selected combinations investigated in this study. Using CompuSyn software, we found that combination treatments of PTC209 and UNC1999 for 72 hours had synergistic effects (combination index $(\mathrm{CI})<0.8$ ) in the MM INA-6 cell line at all concentrations tested (Table 1). In the MM cell line JJN3, we found that combinations of PTC-209 and UNC1999 demonstrated both synergistic $(\mathrm{CI}<0.8)$ and additive (CI between 0.8-1.2) effects (Table 1). PTC-209 and UNC1999 combinations had mainly additive effects (CI between 0.8-1.2) in the RPMI-8226 and LP-1 cell lines, with some combinations showing slight antagonistic effects (Table 1). The recent finding by Bolomsky et al. [34] showing that PTC-209 reduced the expression of MM survival genes such as c-MYC urged us to evaluate the efficacy of PTC-209 as anti-MM agent also in combination with the BET bromodomains inhibitor JQ1. We found that the combined treatment of PTC-209 and JQ1 induced a significant reduction in cell viability over single agent treatment in INA-6, JJN3, RPMI-8226 and LP-1 MM cell lines (Figure 3B). Combinations of PTC209 and JQ1 demonstrated synergistic effects $(\mathrm{CI}<0.8)$ in the INA-6 cell line (Table 2). In JJN-3 and RPMI-8226 PTC-209 and JQ1 combinations showed synergistic (CI < 0.8 ) and additive (CI between 0.8-1.2) anti-MM effects, while mainly additive effects (CI between $0.8-1.2$ ) were observed in the MM cell line LP-1 (Table 2).
A

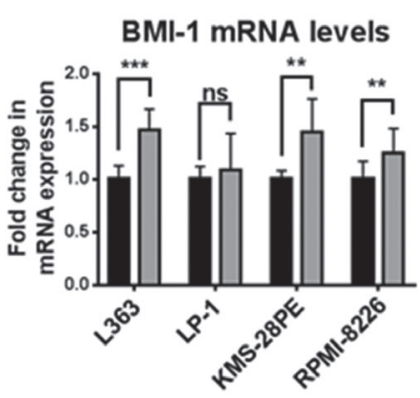

B

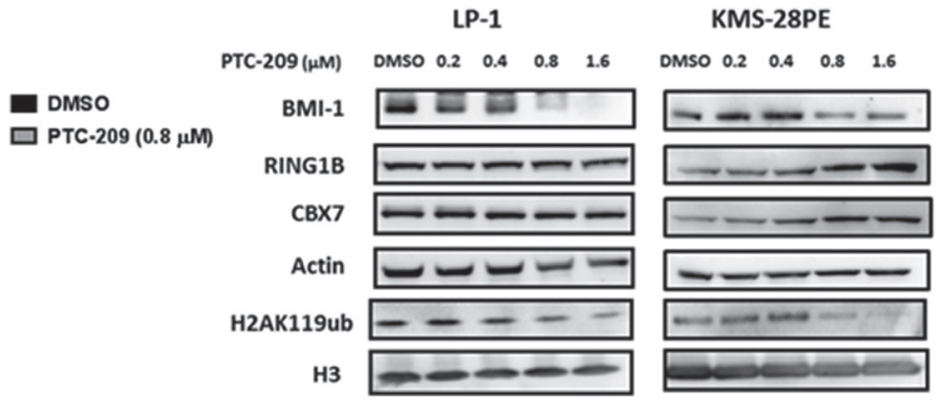

RPMI-8226

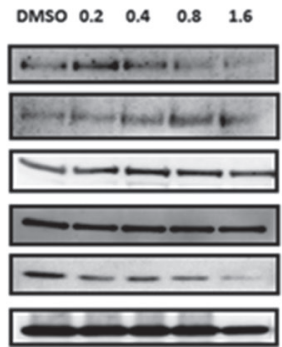

Figure 2: PTC-209 demonstrates on-target effects in MM via downregulation of BMI-1 protein and H2AK119ub levels. (A) PTC-209 treatment does not reduce the BMI-1 transcript levels. Analysis of BMI-1 mRNA levels by RT-qPCR in MM cell lines. Cells were treated with DMSO or $0.8 \mu \mathrm{M}$ of PTC-209 for 48 hours. Changes in mRNA expression were represented relative to DMSO-treated cells. Actin was used as reference gene. Error bars represent standard deviation of three independent biological experiments. $P$-values were calculated using student $t$-test in GraphPad Prism, $P$-value: ${ }^{*} \leq 0.05 ;{ }^{* *} \leq 0.01 ;{ }^{* * *} \leq 0.001$. (B) PTC-209 treatment reduces BMI-1 protein and H2AK119ub global levels in MM cell lines. Analysis of PTC-209 impact on protein levels of PRC1 subunits: BMI-1, CBX-7, RING1B and the associated repressive histone mark H2AK119ub in MM cell lines. Cells were treated with a range of PTC-209 concentrations for 48 hours. DMSO was used as control treatment. Actin and total histone H3 were used as loading controls. Western blots are representative of three independent biological experiments. 


\section{PTC-209 has synergistic and additive activity with UNC1999 and JQ1 in primary MM cells}

Having shown that PTC-209 possesses synergistic and additive effects when combined with UNC1999 or JQ1 in MM cell lines, we sought to investigate whether PTC209 combinatorial effects with UNC1999 or JQ1 could be recapitulated on $\mathrm{CD} 138^{+}$primary cells purified from $\mathrm{MM}$ patients. Indeed we found that combinations of PTC209 with either UNC1999 (Figure 4A) or JQ1 (Figure 4B) reduced the viability of primary MM cells compared with single agent treatment. Similar to combinations in MM cell lines, we observed that combinations of PTC-209 with UNC1999 or JQ1 had synergistic $(\mathrm{CI}<0.8)$ and additive (CI between 0.8-1.2) effects when combined on primary MM cells.

\section{DISCUSSION}

Despite encouraging reports that current treatment strategies can improve multiple myeloma (MM) management, the disease remains fatal [1-4]. Therefore, identification of new therapeutic strategies is imperative for improved MM treatment. Recently, we and others have revealed the importance of epigenetic mechanisms as contributing factors in the development of MM [18, $37,42,47-53]$. The recent success in the development of selective small-molecule inhibitors targeting epigenetic modifiers may now have the potential to increase our understanding of aberrant epigenetic gene regulation in cancer and how inhibiting these components could be utilized for future treatment of patients including $\mathrm{MM}$ $[54,55]$. Among epigenetic modifiers, the BMI-1 protein of the PRC 1 complex now represents a promising target for therapy in MM. This notion is partly based on the fact that BMI-1 is overexpressed in MM during disease progression and relapse [34, 36-38]. Underlining the clinical relevance, BMI-1 overexpression was also found to correlate with poor outcome in refractory MM patients treated with bortezomib or dexamethasone [34]. In 2010, Jagani et al. reported on the importance of BMI-1 for $\mathrm{MM}$ growth in vitro and in vivo by shRNA-mediated knockdown of BMI-1 [37]. The recent development of PTC-209, a selective small-molecule inhibitor of BMI1 , has initiated the possibility to evaluate the therapeutic potential of BMI-1 in tumors including MM [31].

In this study we evaluated the anti-myeloma activity of PTC-209 on a panel of authenticated MM cell lines. We found that PTC-209 demonstrates potent antimyeloma effects in all MM cell lines used in this study. We showed that PTC-209 induced apoptosis in MM cell lines, as determined by an increase in the percentage of Annexin V/PI positive cells and caspase-3/7 activity. This is in agreement with the findings of Bolomsky et al. [34]. Previously, BMI-1 has been suggested to support MM cell growth via direct repression of the pro-apoptotic gene BIM and BMI-1 knockdown reactivated the expression of BIM resulting in MM cell death [37]. In the present study,
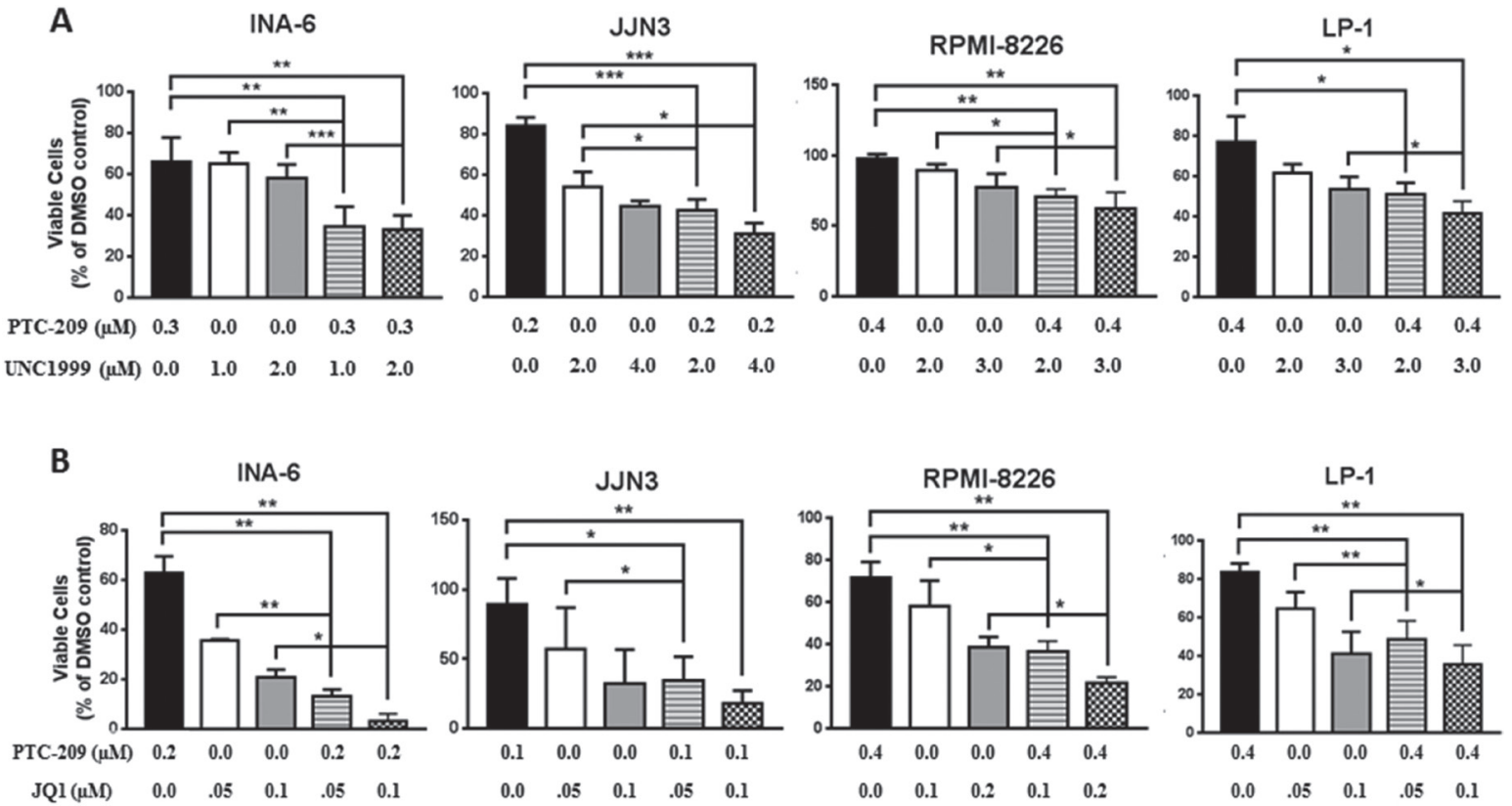

Figure 3: PTC-209 shows synergistic and additive effects with UNC1999 and JQ1 in MM cell lines. Synergistic and/or additive activity of drug combinations was confirmed by co-treatment of MM cell lines with PTC-209 and either UNC1999 (A) or JQ1 (B) at varying concentrations. Combination index $(\mathrm{CI})$ values were determined using CompuSyn software. CI values $<0.8 ; 0.8-1.2$ or $>1.2$ indicated synergistic, additive, or antagonistic drug activities, respectively. DMSO was used as control treatment. Combination treatments were performed for 72 hours. Error bars represent standard deviation of three independent biological experiments. $P$-values were calculated using student $t$-test in GraphPad Prism, $P$-value: ${ }^{*} \leq 0.05 ;{ }^{* *} \leq 0.01 ;{ }^{* * *} \leq 0.001$. 
Table 1: PTC-209 and UNC1999 combination treatments, inhibition rate and combination index (CI)

\begin{tabular}{|c|c|c|c|c|}
\hline Cell Line & PTC-209 $(\mu \mathrm{M})$ & UNC1999 $(\mu \mathrm{M})$ & Inhibition Rate & Combination Index (CI) \\
\hline INA-6 & $\begin{array}{l}0.1 \\
0.1 \\
0.2 \\
0.2 \\
0.3 \\
0.3\end{array}$ & $\begin{array}{l}1 \\
2 \\
1 \\
2 \\
1 \\
2\end{array}$ & $\begin{array}{l}0.44415 \\
0.49388 \\
0.46436 \\
0.50582 \\
0.65551 \\
0.66991\end{array}$ & $\begin{array}{l}0.57996 \\
0.64551 \\
0.68419 \\
0.75362 \\
0.38245 \\
0.40216\end{array}$ \\
\hline JJN3 & $\begin{array}{l}0.1 \\
0.1 \\
0.2 \\
0.2 \\
0.4 \\
0.4\end{array}$ & $\begin{array}{l}2 \\
4 \\
2 \\
4 \\
2 \\
4\end{array}$ & $\begin{array}{l}0.47870 \\
0.78551 \\
0.57284 \\
0.68961 \\
0.76196 \\
0.94817\end{array}$ & $\begin{array}{l}1.22874 \\
0.39410 \\
1.09227 \\
0.92479 \\
1.14930 \\
0.69431\end{array}$ \\
\hline RPMI-8226 & $\begin{array}{l}0.2 \\
0.2 \\
0.4 \\
0.4 \\
0.6 \\
0.6\end{array}$ & $\begin{array}{l}2 \\
3 \\
2 \\
3 \\
2 \\
3\end{array}$ & $\begin{array}{l}0.19562 \\
0.33201 \\
0.29207 \\
0.37471 \\
0.79527 \\
0.81141\end{array}$ & $\begin{array}{l}1.11126 \\
1.13197 \\
1.26375 \\
1.37317 \\
0.86478 \\
0.94670\end{array}$ \\
\hline LP-1 & $\begin{array}{l}0.2 \\
0.2 \\
0.4 \\
0.4 \\
0.6 \\
0.6\end{array}$ & $\begin{array}{l}2 \\
3 \\
2 \\
3 \\
2 \\
3\end{array}$ & $\begin{array}{l}0.50284 \\
0.50177 \\
0.48820 \\
0.58624 \\
0.75672 \\
0.77391\end{array}$ & $\begin{array}{l}0.94805 \\
1.23164 \\
1.39678 \\
1.24663 \\
0.93147 \\
0.95192\end{array}$ \\
\hline
\end{tabular}

Combination index (CI) values were determined using CompuSyn software (ComboSyn, Inc., Paramus, NJ). CI values $<0.8 ; 0.8-1.2$ or $>1.2$ indicated synergistic, additive, or antagonistic drug activities, respectively.

Table 2: PTC-209 and JQ1 combination treatments, inhibition rate and combination index (CI)

\begin{tabular}{|c|c|c|c|c|}
\hline Cell Line & PTC-209 $(\mu \mathrm{M})$ & JQ1 $(\mu \mathrm{M})$ & Inhibition Rate & Combination Index (CI) \\
\hline INA-6 & $\begin{array}{l}0.1 \\
0.1 \\
0.2 \\
0.2 \\
0.3 \\
0.3\end{array}$ & $\begin{array}{c}0.05 \\
0.1 \\
0.05 \\
0.1 \\
0.05 \\
0.1 \\
\end{array}$ & $\begin{array}{l}0.91980 \\
0.92099 \\
0.86716 \\
0.96916 \\
0.95350 \\
0.93535\end{array}$ & $\begin{array}{l}0.28170 \\
0.40982 \\
0.63779 \\
0.27303 \\
0.41319 \\
0.60423\end{array}$ \\
\hline JJN3 & $\begin{array}{l}0.1 \\
0.1 \\
0.2 \\
0.2 \\
0.4 \\
0.4 \\
\end{array}$ & $\begin{array}{c}0.05 \\
0.1 \\
0.05 \\
0.1 \\
0.05 \\
0.1 \\
\end{array}$ & $\begin{array}{l}0.65742 \\
0.82372 \\
0.56127 \\
0.79496 \\
0.73771 \\
0.81171 \\
\end{array}$ & $\begin{array}{l}0.69484 \\
0.67699 \\
1.11905 \\
0.86991 \\
0.91708 \\
1.00260 \\
\end{array}$ \\
\hline RPMI-8226 & $\begin{array}{l}0.2 \\
0.2 \\
0.4 \\
0.4 \\
0.6 \\
0.6 \\
\end{array}$ & $\begin{array}{l}0.1 \\
0.2 \\
0.1 \\
0.2 \\
0.1 \\
0.2 \\
\end{array}$ & $\begin{array}{l}0.52490 \\
0.73503 \\
0.63731 \\
0.78667 \\
0.94489 \\
0.96883 \\
\end{array}$ & $\begin{array}{l}1.13203 \\
0.92359 \\
1.20221 \\
1.03513 \\
0.52199 \\
0.43832 \\
\end{array}$ \\
\hline LP-1 & $\begin{array}{l}0.2 \\
0.2 \\
0.4 \\
0.4 \\
0.6 \\
0.6\end{array}$ & $\begin{array}{c}0.05 \\
0.1 \\
0.05 \\
0.1 \\
0.05 \\
0.1\end{array}$ & $\begin{array}{l}0.45307 \\
0.62035 \\
0.51424 \\
0.64495 \\
0.80423 \\
0.84470\end{array}$ & $\begin{array}{l}1.10495 \\
1.17057 \\
1.25665 \\
1.32982 \\
0.71373 \\
0.80021\end{array}$ \\
\hline
\end{tabular}

Combination index (CI) values were determined using CompuSyn software (ComboSyn, Inc., Paramus, NJ). CI values $<0.8 ; 0.8-1.2$ or $>1.2$ indicated synergistic, additive, or antagonistic drug activities, respectively. 
we evaluated the impact of PTC-209 on the expression of BIM and found that PTC-209 indeed increased the levels of BIM transcripts 48 hours post-treatment in MM cell lines. BIM upregulation was detected as early as 12 hours post-treatment with PTC-209, which coincided with BMI-1 protein downregulation, in the RPMI-8226 cell line (data not shown). This is in contrast to Bolomsky et al. who have reported that PTC-209 had no impact on BIM expression and suggested that PTC-209-mediated apoptosis in MM was likely a consequence of the upregulation of NOXA and downregulation of the antiapoptotic MCL-1 [34]. We speculate that the discrepancy in the results concerning the impact of PTC-209 treatment on BIM expression between our study and Bolomsky et al. [34] might be due to the differences in the choice of time points at which BIM expression was evaluated. The data presented in this study demonstrates that PTC209-induced apoptosis in MM may partly be mediated by direct upregulation of the pro-apoptotic gene BIM. This is in accordance with Jagani et al. showing that BIM knockdown may protect MM cells from the antiMM phenotype mediated by BMI-1 knockdown [37]. In addition, our current study also explores the anti-myeloma activity of PTC-209 on primary MM cells isolated from both newly diagnosed and relapsed patients. Notably, we found that PTC-209 treatment reduced the viability of the majority of MM primary cells derived from both newly diagnosed and relapsed patients. Resistance of cancer cells to treatment and subsequent disease relapse have in some tumors been attributed to a small population of cells with stemness properties [56-58]. Interestingly, BMI-1 expression was found to be upregulated in relapsed MM [34]. As BMI-1 has been assigned an important role in conferring stemness to tumor cells in various types of cancer [31-33], we suggest that targeting BMI-1 in relapsed cancer patients including MM could represent a potential therapeutic strategy. The possibility that BMI-1 would confer stemness characteristics to MM cells is an interesting concept that warrants further investigation.

PTC-209 was initially found to reduce the BMI1 expression in the human colorectal HCT116 cell line [31]. In this study, we showed that PTC-209 treatment in $\mathrm{MM}$ resulted in the downregulation of BMI-1 protein, but not downregulation of transcript levels. This is in agreement with the recent finding showing that PTC-209 did not affect BMI-1 transcript levels but downregulated
A

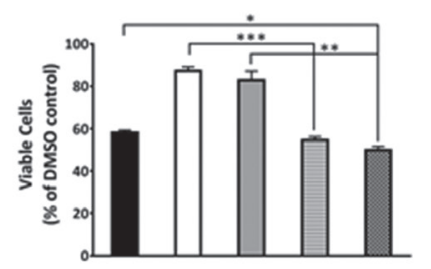

$\begin{array}{rrrrrr}\text { PTC-209 }(\mu \mathrm{M}) & 1.0 & 0.0 & 0.0 & 1.0 & 1.0 \\ \text { UNC1999 }(\mu \mathrm{M}) & 0.0 & 3.0 & 4.0 & 3.0 & 4.0 \\ & & & & \\ \text { Combination Index (CI) } & & 1.27 & 1.21\end{array}$

B
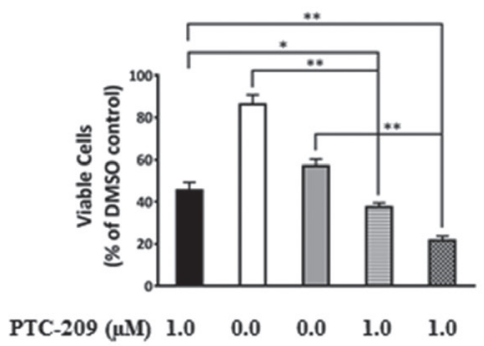

$\begin{array}{llllll}\mathrm{JQ1}(\mu \mathrm{M}) & 0.0 & 0.1 & 0.5 & 0.1 & 0.5\end{array}$

Combination Index (CI)
Patient 1 $0.85 \quad 0.74$
Patient 2

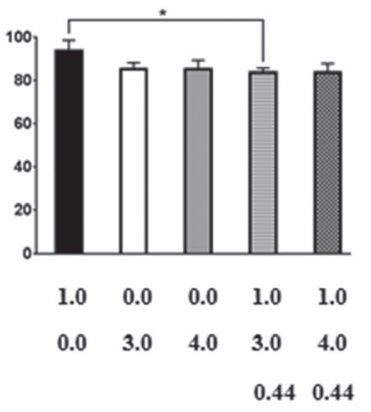

Patient 2

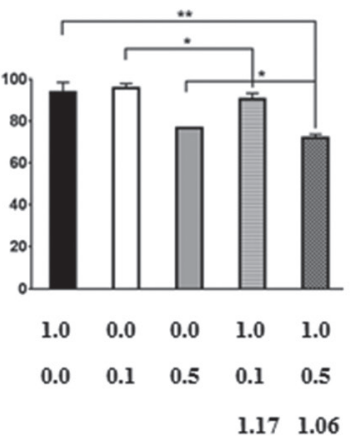

Patient 3

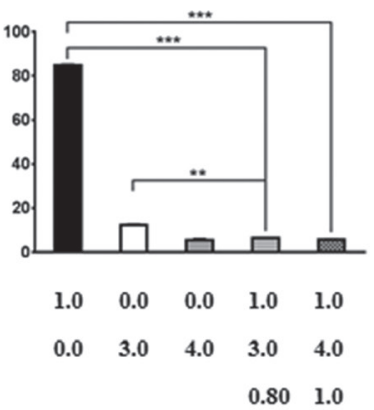

Patient 3

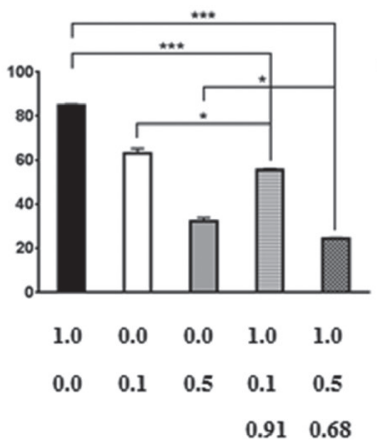

Patient 4

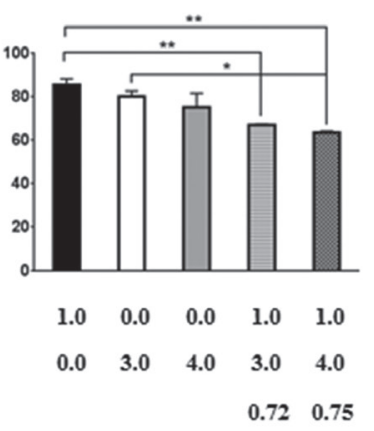

Patient 4

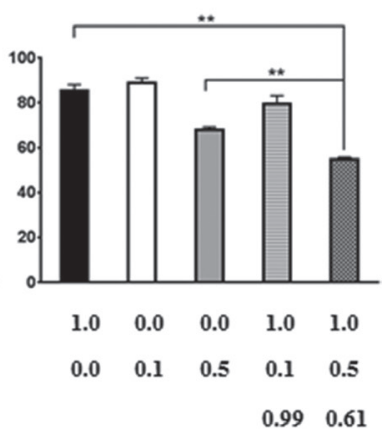

Figure 4: PTC-209 shows synergistic and additive effects with UNC1999 and JQ1 in patient derived-MM cells. Synergistic and/or additive activity of drug combinations was confirmed by co-treatment of CD138 ${ }^{+}$cells purified from newly diagnosed MM patients with PTC-209 and either UNC1999 (A) or JQ1 (B). Combination index (CI) values were determined using CompuSyn software. CI values $<0.8 ; 0.8-1.2$ or $>1.2$ indicated synergistic, additive, or antagonistic drug activities, respectively. DMSO was used as control treatment. Combinatorial treatments were performed for 72 hours. Error bars represent standard deviation of three technical replicates. $P$-values were calculated using student $t$-test in GraphPad Prism, $P$-value: ${ }^{*} \leq 0.05 ;{ }^{* *} \leq 0.01 ;{ }^{* * *} \leq 0.001$. 
BMI-1 protein levels in biliary tract cancer cells [32]. Our data thus suggest that PTC-209 regulates BMI-1 posttranscriptionally. Recent findings using PTC596 to inhibit BMI-1 have suggested protein degradation pathways as underlying mechanisms of drug action [59]. In this study, we found that proteasome inhibition using MG132 in RPMI-8226 and LP-1 cell lines did not counteract PTC209 phenotype i.e. rescuing the BMI-1 protein levels and cell viability (data not shown), which may indicate that PTC-209 is an inhibitor of BMI-1 translation. We further investigated the on-target effects of PTC-209 in MM and evaluated the impact of PTC-209 on other subunits of the PRC1 complex. We could show that PTC-209 neither affected the enzymatic E3 ubiquitin ligase RING1 subunit nor other core subunits such as CBX-7. PTC209-mediated downregulation of BMI-1 led to global reduction in the $\mathrm{H} 2 \mathrm{AK} 119 \mathrm{ub}$ histone mark, consistent with reduced ubiquitin ligase activity of PRC1. These data are in agreement with the initial finding reporting BMI-1 to be a target of PTC-209 [31]. The demonstration of on-target effects of PTC-209 in MM thus suggests chromatin-mediated mechanisms as important mediators underpinning its anti-MM activity.

Recently, PTC-209 was shown to have synergistic and/or additive effects when combined with dexamethasone, pomalidomide and carfilzomib in MM [34]. Supporting the use of pharmacological inhibition of BMI-1 in combinatorial regimens, shRNA-mediated knockdown of BMI-1 was found to sensitize MM cells to bortezomib treatment [60]. To further study the potential use of PTC-209 as an anti-MM agent, we investigated the anti-MM effects of PTC-209 in combinatorial epigenetic regimens in $\mathrm{MM}$ cell lines and malignant $\mathrm{CD}_{138^{+}}$primary cells. PRC1 and PRC2 co-operation in gene silencing has been suggested during normal development and tumorigenesis [39-41]. We and others have recently shown that $\mathrm{EZH} 2$ inhibition demonstrates anti-MM effects using in vitro and in vivo models [4246]. Therefore, combinatorial inhibition of PRC1 and PRC2 is indeed of interest in MM. We used PTC-209 and the EZH2 inhibitor UNC1999 to assess the effects of single and combinatorial treatments on the viability of MM cell lines and primary cells in vitro. We found that combinations of PTC-209 and UNC1999 have synergistic and/or additive effects in reducing the viability of MM cells. Recently, PTC-209 was shown to downregulate the expression of MM survival genes such as c-MYC, MCL1 and CCND1 [34]. Deregulation of c-MYC has been suggested to be an important step in disease progression from the asymptomatic monoclonal gammopathy of undetermined significance (MGUS) stage to the symptomatic MM in human [61] and in murine models [62]. Furthermore, targeted inhibition of c-MYC and its expression signature by BET bromodomains inhibitors has been proven to be a potential therapeutic strategy in
MM [63-65]. Taken together, these findings prompted us to evaluate the combination of PTC-209 and JQ1 on the viability of MM cell lines. Indeed, we found that PTC209 and JQ1 demonstrated synergistic and/or additive effects when combined in MM cell lines and primary cells. Collectively, the data presented in this study further support BMI-1 as a candidate for targeted therapy in $\mathrm{MM}$, but also propose combinatorial epigenetic therapy as a novel treatment in MM.

In conclusion, the present study further emphasizes that the BMI-1 inhibitor PTC-209 has potent anti-MM activity and supports the notion that BMI-1 may be a potential therapeutic target in novel MM treatment strategies. We present epigenetic inhibitors as a novel therapeutic intervention for MM by further highlighting the potential use of BMI-1 inhibitors alone or in combination with other epigenetic inhibitors directed to $\mathrm{PRC} 2 / \mathrm{EZH} 2$ or BET bromodomains in targeting the malignant MM cell.

\section{MATERIALS AND METHODS}

\section{Cell culture and treatment}

All MM authenticated cell lines [66] used in this study were maintained in RPMI-1640 AQmediaTM (Sigma) supplemented with 10\% FBS (Sigma), 1\% GlutaMax $^{\mathrm{TM}}$ (Gibco) and antibiotics (penicillin $100 \mathrm{U} /$ $\mathrm{mL}$ and streptomycin $50 \mathrm{mg} / \mathrm{mL}$; Sigma) at $37^{\circ} \mathrm{C}$ in a humidified $5 \% \mathrm{CO}_{2}$ in-air atmosphere. The INA-6 and U1996 cell lines were cultured in the presence of Interleukin-6 (IL-6). Exponentially growing cells were seeded at 200000 cells $/ \mathrm{mL}$ overnight before addition of reagents.

\section{Reagents}

The BMI-1 inhibitor PTC-209 was purchased from (Sigma Aldrich, CAS \#: 315704-66-6). The BET bromodomain inhibitor JQ1 was purchased from (BPS Bioscience, Catalog \#: 27400). UNC1999 was synthesized according to previously published procedures [67].

\section{Cell viability assay for MM cell lines}

MM cell lines were seeded in 12-well plate at 200000 cells $/ \mathrm{ml}$ overnight then treated with a range of PTC-209 concentrations for 48 hours with DMSO used as control treatment. On the day of analysis, cells were seeded in triplicate wells in 96-well flat-bottom plates. Cell viability was assessed using Resazurin assay reduction method using AlamarBlue (Sigma-Aldrich) as previously described [18]. Fluorescence level of at least 5 times of the blank was set as threshold for DMSO control treated sample to be included in the analysis. 


\section{Patients and patient material}

The ethics committees of the participating hospitals approved the study and patient samples obtained following informed consent in compliance with the Declaration of Helsinki. A total of 11 bone marrow (BM) aspirates were collected from 4 diagnostic and 7 relapsed myeloma patients. Patient characteristics are detailed in Supplementary Table 1. For drug combination experiments, heparinized bone marrow samples were obtained from newly diagnosed MM patients in accordance with the Declaration of Helsinki and approved by the local ethics committees of Uppsala and Stockholm (Dnr 2004:M-332 and 2010/1478-32).

\section{Cytogenetics}

Plasma cells were selected by immunomagnetic enrichment for $\mathrm{CD}_{138^{+}}$cells (Human Whole Blood CD138 Microbeads Column Kit, Miltenyi Biotec, Bergisch Gladbach, Germany). Selected cells $(n \geq 100)$ were used for interphase fluorescence in situ hybridization (FISH) following the guidelines of the European Myeloma Network 2012 [68]. The probes used for FISH were described previously [69].

\section{Drug sensitivity and resistance testing}

CD138 $8^{+}$cells were enriched with the EasySep ${ }^{\mathrm{TM}}$ Human CD138 Positive Selection Kit (StemCell Technologies, Grenoble, France) using the mononuclear cell fraction of BM aspirates following gradient separation (Ficoll-Paque PREMIUM; GE Healthcare, Little Chalfont, Buckinghamshire, UK). $\mathrm{CD} 138^{+}$cells derived from myeloma patients with $90 \%$ purity were tested against PTC-209 in 5 concentrations in 10-fold dilutions covering a 10,000-fold concentration range (1-10,000 nM). In brief, $5 \mu \mathrm{L}$ of a $5 \times$ PTC-209 solution was diluted in cell culture medium comprised of RPMI-1640 medium supplemented with $10 \%$ fetal bovine serum, $2 \mathrm{mM}$ L-glutamine, penicillin $(100 \mathrm{U} / \mathrm{mL})$, streptomycin $(100 \mu \mathrm{g} / \mathrm{mL})$ and $25 \%$ conditioned medium from the HS-5 human bone marrow stromal cell line [70] and added to 384-well drug plates. $\mathrm{CD} 138^{+}$cells were diluted in the cell culture medium and $20 \mu \mathrm{L}$ of the cell suspension containing 5000 cells was transferred to each well using a MultiDrop Combi peristaltic dispenser (Thermo Scientific, Waltham, MA, USA). The plates were incubated in a humidified environment at $37^{\circ} \mathrm{C}$ and $5 \% \mathrm{CO}_{2}$. Cell viability was measured after $72 \mathrm{~h}$ using the CellTiter-Glo assay (Promega, Madison, WI, USA) with a PHERAstar ${ }^{\circledR}$ microplate reader to measure luminescence (BMG-Labtech, Offenburg, Germany). The data was normalized to untreated cells (DMSO only). Results from triplicate screens were used to generate 5-point doseresponse curves in Graph Pad Prism version 7.00 for MAC OS, GraphPad Software, La Jolla California USA.

\section{Flow cytometric analysis}

Cells were seeded at 200000 cells $/ \mathrm{mL}$ overnight before addition of reagents then were cultured for 48 hours in the presence of DMSO control or $0.8 \mu \mathrm{M}$ of PTC-209. Apoptosis was quantified by Annexin V (AV)fluorescein isothocyanate (FITC) using TACS Annexin V-FITC Apoptosis Kit (R\&D Systems, Gaithersburg, MD, USA). Samples were treated according to manufacturer's recommendations and analyzed by flow cytometry (FACScan), presenting apoptotic cells as Annexin V-positive/PI-negative cells and necrotic cells as Annexin V-positive/PI-positive cells. Cell cycle analysis was performed by quantitation of DNA content using propidium iodide (PI) DNA staining. The distribution of cells in the different cycle phases was performed following the Vindelov method. In short, cells were washed with ice-cold PBS, lysed using NP-40 buffer and trypsin $(0.03 \mathrm{mg} / \mathrm{mL})$ for 10 minutes at room temperature. Next, samples were incubated in trypsin inhibitor $(0.5 \mathrm{mg} / \mathrm{mL})$ and ribonuclease $\mathrm{A}(0.1 \mathrm{mg} / \mathrm{mL})$ for 10 minutes at room temperature. DNA staining was performed by addition of PI $(0.42 \mathrm{mg} / \mathrm{mL})$ and incubation for 15 minutes at $4^{\circ} \mathrm{C}$. The modfit LT 3.1 Analysis Software (Verity Software House) was used to calculate the proportion of cycling cells in each of the cell cycle phases.

\section{Protein extraction and western blot}

Following treatment with DMSO or PTC-209 for 48 hours, MM cell lines were harvested, washed with ice-cold PBS and collected at $1500 \mathrm{rpm}$ for 5 minutes. Total cellular protein was extracted using RIPA extraction buffer with freshly added protease inhibitor cocktail. Western blotting was performed as previously described [42]. Histone proteins were extracted using the Episeeker histone extraction kit (abcam, Ab113476) following the manufacturer's procedure. Antibodies against BMI-1 (Santa Cruz, sc-390443), RING1B (ab3832), CBX-7 (abcam, ab21873), Actin (Santa Cruz, sc-1616), H2AK119ub (Millipore) and Histone H3 (abcam, Ab1791) were used in this study.

\section{mRNA expression analysis}

Total RNA was extracted using TRIzol ${ }^{\circledR}$ reagent (Invitrogen) and reverse transcription was performed using random primers (Invitrogen) was performed on $1 \mu \mathrm{g}$ of total RNA using SuperScriptTM III Reverse Transcriptase (Invitrogen) according to the manufacturer's protocol. RTqPCR analysis of mRNA was performed using TaqMan ${ }^{\circledR}$ gene expression assays (Applied Biosystems) for BMI-1 (Assay ID: Hs00995536_m1, Catalog \#: 4448892), BIM (Assay ID: Hs00708019_s1, Catalog \#: 4453320) and Actin (Assay ID: Hs01060665_g1, Catalog \#: 4448892) as housekeeping mRNA. 


\section{Combination treatment and calculation of combination indexes}

The MM cell lines were seeded at 200000 cells $/ \mathrm{ml}$ in 12 well plates overnight before treatment. The INA6 cell line was cultured in the presence of IL-6. Cells were treated with single drugs or combination of drugs for 72 hours. For primary cells, $\mathrm{CD} 138^{+} \mathrm{MM}$ cells were purified from 4 newly diagnosed MM patients using immunomagnetic purification by Whole Blood Column Kit (MACS, Miltenyi Biotec, Paris, France) according to the manufacturer's protocols. Subsequently, the purity of the CD138-enriched fraction was evaluated by MayGrünwald-Giemsa staining (Supplementary Table 2). Cell viability was assessed using AlamarBlue assay (SigmaAldrich) as previously described [18] and as mentioned above. Combination index (CI) was calculated using CompuSyn software (ComboSyn, Inc., Paramus, NJ). CI values $<0.8 ; 0.8-1.2$ or $>1.2$ indicated synergistic, additive, or antagonistic drug activities, respectively.

\section{Statistical analysis}

Paired, two-tailed Student $t$-test was calculated using GraphPad Prism, ${ }^{*}: P$-value $\leq 0.05,{ }^{* *}: P$-value $\leq$ $0.01,{ }^{* * *}: P$-value $\leq 0.001$.

\section{ACKNOWLEDGMENTS}

We are grateful to Charlotta Sandberg Blixt for the excellent technical assistance, to the patients providing primary material, to Drs. Pekka Anttila, Raija Silvennoinen and Juha Lievonen for clinical information, and to the High Throughput Biomedicine Unit at FIMM. FACS analysis was performed at BioVis - Biological Visualization, Science for Life Laboratory, Department of Immunology, Genetics and Pathology, Rudbeck Laboratory, Uppsala University, Sweden.

\section{CONFLICTS OF INTEREST}

The authors declare no conflicts of interest exist.

\section{GRANT SUPPORT}

This work was supported by grants from the Swedish Cancer Society, the Hans von Kantzow Foundation, the Swedish Research Council and grants R01CA218600 and R01GM122749 (to J.J.) from the U.S. National Institutes of Health.

\section{REFERENCES}

1. Raab MS, Podar K, Breitkreutz I, Richardson PG, Anderson KC. Multiple myeloma. Lancet. 2009; 374:324-339.
2. Kumar S. Multiple myeloma-current issues and controversies. Cancer Treat Rev. 2010; 36:S3-11.

3. Palumbo A, Anderson K. Multiple myeloma. N Engl J Med. 2011; 364:1046-1060.

4. Mahindra A, Laubach J, Raje N, Munshi N, Richardson PG, Anderson K. Latest advances and current challenges in the treatment of multiple myeloma. Nat Rev Clin Oncol. 2012; 9:135-143.

5. Joao C, Costa C, Coelho I, Vergueiro MJ, Ferreira M, da Silva MG. Long-term survival in multiple myeloma. Clin Case Rep. 2014; 2:173-179.

6. Abdi J, Chen G, Chang H. Drug resistance in multiple myeloma: latest findings and new concepts on molecular mechanisms. Oncotarget. 2013; 4:2186-2207. https://doi. org/10.18632/oncotarget.1497.

7. Bianchi G, Munshi NC. Pathogenesis beyond the cancer clone(s) in multiple myeloma. Blood. 2015; 125:30493058.

8. Kim JK, Samaranayake M, Pradhan S. Epigenetic mechanisms in mammals. Cell Mol Life Sci. 2009; 66:596-612.

9. Goldberg AD, Allis CD, Bernstein E. Epigenetics: a landscape takes shape. Cell. 2007; 128:635-638.

10. Dawson MA, Kouzarides T. Cancer epigenetics: from mechanism to therapy. Cell. 2012; 150:12-27.

11. Wilting RH, Dannenberg JH. Epigenetic mechanisms in tumorigenesis, tumor cell heterogeneity and drug resistance. Drug Resist Updat. 2012; 15:21-38.

12. Plass C, Pfister SM, Lindroth AM, Bogatyrova O, Claus $\mathrm{R}$, Lichter P. Mutations in regulators of the epigenome and their connections to global chromatin patterns in cancer. Nat Rev Genet. 2013; 14:765-780.

13. Chapman MA, Lawrence MS, Keats JJ, Cibulskis K, Sougnez C, Schinzel AC, Harview CL, Brunet JP, Ahmann GJ, Adli M, Anderson KC, Ardlie KG, Auclair D, et al. Initial genome sequencing and analysis of multiple myeloma. Nature. 2011; 471:467-472.

14. Pawlyn C, Kaiser MF, Heuck C, Melchor L, Wardell CP, Murison A, Chavan SS, Johnson DC, Begum DB, Dahir NM, Proszek PZ, Cairns DA, Boyle EM, et al. The Spectrum and Clinical Impact of Epigenetic Modifier Mutations in Myeloma. Clin Cancer Res. 2016; 22:5783-5794.

15. Dupere-Richer D, Licht JD. Epigenetic regulatory mutations and epigenetic therapy for multiple myeloma. Curr Opin Hematol. 2017; 24:336-344.

16. Zhan F, Hardin J, Kordsmeier B, Bumm K, Zheng M, Tian E, Sanderson R, Yang Y, Wilson C, Zangari M, Anaissie E, Morris C, Muwalla F, et al. Global gene expression profiling of multiple myeloma, monoclonal gammopathy of undetermined significance, and normal bone marrow plasma cells. Blood. 2002; 99:1745-1757.

17. Croonquist PA, Van Ness B. The polycomb group protein enhancer of zeste homolog 2 (EZH 2) is an oncogene that influences myeloma cell growth and the mutant ras phenotype. Oncogene. 2005; 24:6269-6280. 
18. Kalushkova A, Fryknas M, Lemaire M, Fristedt C, Agarwal P, Eriksson M, Deleu S, Atadja P, Osterborg A, Nilsson K, Vanderkerken K, Oberg F, Jernberg-Wiklund H. Polycomb target genes are silenced in multiple myeloma. PLoS One. 2010; 5:e11483.

19. Keats JJ, Maxwell CA, Taylor BJ, Hendzel MJ, Chesi M, Bergsagel PL, Larratt LM, Mant MJ, Reiman T, Belch AR, Pilarski LM. Overexpression of transcripts originating from the MMSET locus characterizes all $\mathrm{t}(4 ; 14)(\mathrm{p} 16 ; \mathrm{q} 32)-$ positive multiple myeloma patients. Blood. 2005; 105:4060-4069.

20. Popovic R, Martinez-Garcia E, Giannopoulou EG, Zhang Q, Zhang Q, Ezponda T, Shah MY, Zheng Y, Will CM, Small EC, Hua Y, Bulic M, Jiang Y, et al. Histone Methyltransferase MMSET/NSD2 Alters EZH2 Binding and Reprograms the Myeloma Epigenome through Global and Focal Changes in H3K36 and H3K27 Methylation. PLoS Genet. 2014; 10:e1004566.

21. Ohguchi H, Harada T, Sagawa M, Kikuchi S, Tai YT, Richardson PG, Hideshima T, Anderson KC. KDM6B modulates MAPK pathway mediating multiple myeloma cell growth and survival. Leukemia. 2017. [Epub ahead of print].

22. Ohguchi H, Hideshima T, Bhasin MK, Gorgun GT, Santo L, Cea M, Samur MK, Mimura N, Suzuki R, Tai YT, Carrasco RD, Raje N, Richardson PG, et al. The KDM3A-KLF2IRF4 axis maintains myeloma cell survival. Nat Commun. 2016; 7:10258.

23. Aloia L, Di Stefano B, Di Croce L. Polycomb complexes in stem cells and embryonic development. Development. 2013; 140:2525-2534.

24. Sauvageau M, Sauvageau G. Polycomb group proteins: multi-faceted regulators of somatic stem cells and cancer. Cell Stem Cell. 2010; 7:299-313.

25. Di Croce L, Helin K. Transcriptional regulation by Polycomb group proteins. Nat Struct Mol Biol. 2013; 20:1147-1155.

26. Aranda S, Mas G, Di Croce L. Regulation of gene transcription by Polycomb proteins. Sci Adv. 2015; 1:e1500737.

27. Bhattacharya R, Mustafi SB, Street M, Dey A, Dwivedi SK. Bmi-1: At the crossroads of physiological and pathological biology. Genes Dis. 2015; 2:225-239.

28. van Lohuizen M, Verbeek S, Scheijen B, Wientjens E, van der Gulden H, Berns A. Identification of cooperating oncogenes in $\mathrm{E}$ mu-myc transgenic mice by provirus tagging. Cell. 1991; 65:737-752.

29. Haupt Y, Alexander WS, Barri G, Klinken SP, Adams JM. Novel zinc finger gene implicated as myc collaborator by retrovirally accelerated lymphomagenesis in E mu-myc transgenic mice. Cell. 1991; 65:753-763.

30. Sahasrabuddhe AA. BMI1: A Biomarker of Hematologic Malignancies. Biomark Cancer. 2016; 8:65-75.

31. Kreso A, van Galen P, Pedley NM, Lima-Fernandes E, Frelin C, Davis T, Cao L, Baiazitov R, Du W, Sydorenko N, Moon
YC, Gibson L, Wang Y, et al. Self-renewal as a therapeutic target in human colorectal cancer. Nat Med. 2014; 20:29-36.

32. Mayr C, Wagner A, Loeffelberger M, Bruckner D, Jakab M, Berr F, Di Fazio P, Ocker M, Neureiter D, Pichler M, Kiesslich T. The BMI1 inhibitor PTC-209 is a potential compound to halt cellular growth in biliary tract cancer cells. Oncotarget. 2016; 7:745-758. https://doi. org/10.18632/oncotarget.6378.

33. Raaphorst FM. Self-renewal of hematopoietic and leukemic stem cells: a central role for the Polycomb-group gene Bmi1. Trends Immunol. 2003; 24:522-524.

34. Bolomsky A, Schlangen K, Schreiner W, Zojer N, Ludwig H. Targeting of BMI-1 with PTC-209 shows potent anti-myeloma activity and impairs the tumour microenvironment. J Hematol Oncol. 2016; 9:17.

35. Darwish NH, Sudha T, Godugu K, Elbaz O, Abdelghaffar HA, Hassan EE, Mousa SA. Acute myeloid leukemia stem cell markers in prognosis and targeted therapy: potential impact of BMI-1, TIM-3 and CLL-1. Oncotarget. 2016; 7:57811-57820. https://doi.org/10.18632/oncotarget.11063.

36. Zhan F, Barlogie B, Arzoumanian V, Huang Y, Williams DR, Hollmig K, Pineda-Roman M, Tricot G, van Rhee F, Zangari M, Dhodapkar M, Shaughnessy JD Jr. Geneexpression signature of benign monoclonal gammopathy evident in multiple myeloma is linked to good prognosis. Blood. 2007; 109:1692-1700.

37. Jagani Z, Wiederschain D, Loo A, He D, Mosher R, Fordjour P, Monahan J, Morrissey M, Yao YM, Lengauer C, Warmuth M, Sellers WR, Dorsch M. The Polycomb group protein Bmi-1 is essential for the growth of multiple myeloma cells. Cancer Res. 2010; 70:5528-5538.

38. Chng WJ, Kumar S, Vanwier S, Ahmann G, Price-Troska T, Henderson K, Chung TH, Kim S, Mulligan G, Bryant B, Carpten J, Gertz M, Rajkumar SV, et al. Molecular dissection of hyperdiploid multiple myeloma by gene expression profiling. Cancer Res. 2007; 67:2982-2989.

39. Beguelin W, Teater M, Gearhart MD, Calvo Fernandez MT, Goldstein RL, Cardenas MG, Hatzi K, Rosen M, Shen H, Corcoran CM, Hamline MY, Gascoyne RD, Levine RL, et al. EZH2 and BCL6 Cooperate to Assemble CBX8-BCOR Complex to Repress Bivalent Promoters, Mediate Germinal Center Formation and Lymphomagenesis. Cancer Cell. 2016; 30:197-213.

40. Wu X, Gong Y, Yue J, Qiang B, Yuan J, Peng X. Cooperation between EZH2, NSPc1-mediated histone H2A ubiquitination and Dnmt1 in HOX gene silencing. Nucleic Acids Res. 2008; 36:3590-3599.

41. Blackledge NP, Farcas AM, Kondo T, King HW, McGouran JF, Hanssen LL, Ito S, Cooper S, Kondo K, Koseki Y, Ishikura T, Long HK, Sheahan TW, et al. Variant PRC1 complex-dependent H2A ubiquitylation drives PRC2 recruitment and polycomb domain formation. Cell. 2014; 157:1445-1459.

42. Agarwal P, Alzrigat M, Parraga AA, Enroth S, Singh U, Ungerstedt J, Osterborg A, Brown PJ, Ma A, Jin J, Nilsson 
K, Oberg F, Kalushkova A, et al. Genome-wide profiling of histone H3 lysine 27 and lysine 4 trimethylation in multiple myeloma reveals the importance of Polycomb gene targeting and highlights EZH2 as a potential therapeutic target. Oncotarget. 2016; 7:6809-6823. https://doi. org/10.18632/oncotarget.6843.

43. Hernando H, Gelato KA, Lesche R, Beckmann G, Koehr S, Otto S, Steigemann P, Stresemann C. EZH2 Inhibition Blocks Multiple Myeloma Cell Growth through Upregulation of Epithelial Tumor Suppressor Genes. Mol Cancer Ther. 2016; 15:287-298.

44. Pawlyn C, Bright MD, Buros AF, Stein CK, Walters Z, Aronson LI, Mirabella F, Jones JR, Kaiser MF, Walker BA, Jackson GH, Clarke PA, Bergsagel PL, et al. Overexpression of EZH2 in multiple myeloma is associated with poor prognosis and dysregulation of cell cycle control. Blood Cancer J. 2017; 7:e549.

45. Zeng D, Liu M, Pan J. Blocking EZH2 methylation transferase activity by GSK126 decreases stem cell-like myeloma cells. Oncotarget. 2017; 8:3396-3411. https://doi. org/10.18632/oncotarget.13773.

46. Alzrigat M, Parraga AA, Agarwal P, Zureigat H, Osterborg A, Nahi H, Ma A, Jin J, Nilsson K, Oberg F, Kalushkova A, Jernberg-Wiklund H. EZH2 inhibition in multiple myeloma downregulates myeloma associated oncogenes and upregulates microRNAs with potential tumor suppressor functions. Oncotarget. 2017; 8:10213-10224. https://doi. org/10.18632/onc otarget.14378.

47. Martinez-Garcia E, Popovic R, Min DJ, Sweet SM, Thomas PM, Zamdborg L, Heffner A, Will C, Lamy L, Staudt LM, Levens DL, Kelleher NL, Licht JD. The MMSET histone methyl transferase switches global histone methylation and alters gene expression in $\mathrm{t}(4 ; 14)$ multiple myeloma cells. Blood. 2011; 117:211-220.

48. Furukawa Y, Kikuchi J. Epigenetic mechanisms of cell adhesion-mediated drug resistance in multiple myeloma. Int J Hematol. 2016; 104:281-292.

49. Agirre X, Castellano G, Pascual M, Heath S, Kulis M, Segura V, Bergmann A, Esteve A, Merkel A, Raineri E, Agueda L, Blanc J, Richardson D, et al. Wholeepigenome analysis in multiple myeloma reveals DNA hypermethylation of B cell-specific enhancers. Genome Res. 2015; 25:478-487.

50. Chen L, Li C, Zhang R, Gao X, Qu X, Zhao M, Qiao C, Xu J, Li J. miR-17-92 cluster microRNAs confers tumorigenicity in multiple myeloma. Cancer Lett. 2011; 309:62-70.

51. Chim CS, Liang R, Leung MH, Yip SF, Kwong YL. Aberrant gene promoter methylation marking disease progression in multiple myeloma. Leukemia. 2006; 20:1190-1192.

52. Sive JI, Feber A, Smith D, Quinn J, Beck S, Yong K. Global hypomethylation in myeloma is associated with poor prognosis. Br J Haematol. 2016; 172:473-475.

53. Pichiorri F, Suh SS, Ladetto M, Kuehl M, Palumbo T, Drandi D, Taccioli C, Zanesi N, Alder H, Hagan JP, Munker R,
Volinia S, Boccadoro M, et al. MicroRNAs regulate critical genes associated with multiple myeloma pathogenesis. Proc Natl Acad Sci USA. 2008; 105:12885-12890.

54. Woollard PM, Mehta NA, Vamathevan JJ, Van Horn $\mathrm{S}$, Bonde BK, Dow DJ. The application of nextgeneration sequencing technologies to drug discovery and development. Drug Discov Today. 2011; 16:512-519.

55. Jones PA. At the tipping point for epigenetic therapies in cancer. J Clin Invest. 2014; 124:14-16.

56. Dean M, Fojo T, Bates S. Tumour stem cells and drug resistance. Nat Rev Cancer. 2005; 5:275-284.

57. Di C, Zhao Y. Multiple drug resistance due to resistance to stem cells and stem cell treatment progress in cancer (Review). Exp Ther Med. 2015; 9:289-293.

58. Koren E, Fuchs Y. The bad seed: Cancer stem cells in tumor development and resistance. Drug Resist Updat. 2016; 28:1-12.

59. Kim MJ, Cao L, Sheedy J, Risher N, Dumble M, Lee CS, Sydorenko N, Baiazitov R, Du W, Moon YC, Weetall ML, Colacino J, Davis TW. Abstract 5517: PTC596-induced Bmi1 hyper-phosphorylation via Cdk1/2 activation resulting in tumor stem cell depletion. Cancer Research. 2014; 74:5517-5517.

60. Wu SQ, Xu ZZ, Niu WY, Huang HB, Zhan R. ShRNAmediated Bmi-1 silencing sensitizes multiple myeloma cells to bortezomib. Int J Mol Med. 2014; 34:616-623.

61. Chng WJ, Huang GF, Chung TH, Ng SB, Gonzalez-Paz N, Troska-Price T, Mulligan G, Chesi M, Bergsagel PL, Fonseca R. Clinical and biological implications of MYC activation: a common difference between MGUS and newly diagnosed multiple myeloma. Leukemia. 2011; 25:1026-1035.

62. Chesi M, Robbiani DF, Sebag M, Chng WJ, Affer M, Tiedemann R, Valdez R, Palmer SE, Haas SS, Stewart AK, Fonseca R, Kremer R, Cattoretti G, et al. AID-dependent activation of a MYC transgene induces multiple myeloma in a conditional mouse model of post-germinal center malignancies. Cancer Cell. 2008; 13:167-180.

63. Holien T, Sundan A. Oncogene addiction to c-MYC in myeloma cells. Oncotarget. 2012; 3:739-740. https://doi. org/10.18632/oncotarget.631.

64. Holien T, Vatsveen TK, Hella H, Waage A, Sundan A. Addiction to c-MYC in multiple myeloma. Blood. 2012; 120:2450-2453.

65. Delmore JE, Issa GC, Lemieux ME, Rahl PB, Shi J, Jacobs HM, Kastritis E, Gilpatrick T, Paranal RM, Qi J, Chesi M, Schinzel AC, McKeown MR, et al. BET bromodomain inhibition as a therapeutic strategy to target c-Myc. Cell. $2011 ; 146: 904-917$.

66. Jernberg-Wiklund H, Nilsson K. Multiple Myeloma Cell Lines. In: Masters JW and Palsson B, Editors. Human Cell Culture: Springer Netherlands. 2002; pp.81-155.

67. Konze KD, Ma A, Li F, Barsyte-Lovejoy D, Parton T, Macnevin CJ, Liu F, Gao C, Huang XP, Kuznetsova E, Rougie M, Jiang A, Pattenden SG, et al. An orally bioavailable chemical probe of the Lysine 
Methyltransferases EZH2 and EZH1. ACS Chem Biol. 2013; 8:1324-1334.

68. Ross FM, Avet-Loiseau H, Ameye G, Gutierrez NC, Liebisch P, O'Connor S, Dalva K, Fabris S, Testi AM, Jarosova M, Hodkinson C, Collin A, Kerndrup G, et al. Report from the European Myeloma Network on interphase FISH in multiple myeloma and related disorders. Haematologica. 2012; 97:1272-1277.

69. Majumder MM, Silvennoinen R, Anttila P, Tamborero D, Eldfors S, Yadav B, Karjalainen R, Kuusanmaki H,
Lievonen J, Parsons A, Suvela M, Jantunen E, Porkka K, et al. Identification of precision treatment strategies for relapsed/refractory multiple myeloma by functional drug sensitivity testing. Oncotarget. 2017; 8:56338-56350. https://doi.org/10.18632/oncotarget.17630.

70. Roecklein BA, Torok-Storb B. Functionally distinct human marrow stromal cell lines immortalized by transduction with the human papilloma virus E6/E7 genes. Blood. 1995; 85:997-1005. 